\title{
$k$-GROUPS AND DUALITY
}

\author{
BY \\ N. NOBLE( $\left.{ }^{1}\right)$
}

Recall that a function is $k$-continuous if its restriction to each compact subset of its domain is continuous. We call a topological group $G$ a $k$-group if each $k$-continuous homomorphism on $G$ is continuous. As we will see in $\S 1$, where elementary properties of $k$-groups are studied, $k$-groups are the appropriate topological group analogue to $k$-spaces. As one would expect, they provide a useful tool for the study of dual groups topologized with the compact-open topology. In $\$ 2$ we show that the duality map on an abelian $k$-group is always continuous, and in $\$ 3$ we give some extensions of the Pontrjagin-Van Kampen duality theorem. As a corollary we show that each closed subgroup of a countable product of locally compact abelian groups satisfies duality, extending the principal result of [8] - that the inverse limit of a sequence of locally compact abelian groups satisfies duality.

1. Elementary properties. Recall that where $(X, t)$ is a topological space and $k(t)=\{U \subseteq X:$ For each compact $K \subseteq X, U \cap K$ is relatively open in $K\},(X, k(t))$ is a topological space and $k(t)$ is called the $k$-extension of $t$. (Also, when $(X, t)$ is denoted as $X,(X, k(t))$ is denoted as $k X$ and is called the $k$-extension of $X$.) The topology $k(t)$ is the largest topology on $X$ coinciding on compact sets with $t$ and $X$ is called a $k$-space if $X=k X$. Obviously $X$ is a $k$-space if and only if each $k$-continuous function on $X$ is continuous. Where $(G, t)$ is a topoligical group, let $k_{g}(t)$ denote the supremum of all group topologies $t^{\prime}$ satisfying $t^{\prime} \leqq k(t)$; and where $(G, t)$ is denoted as $G$, denote $\left(G, k_{g}(t)\right)$ as $k_{g} G$.

1.1. Proposition. If $(G, t)$ is any topological group, then $k_{g}(t)$ is the largest group topology for $G$ coinciding on compact sets with $t$. Thus $(G, t)$ is a k-group if and only if $k_{g}(t)=t$.

Proof. Since the supremum of any collection of group topologies is a group topology, $k_{g}(t)$ is a group topology; since $t \leqq k_{g}(t) \leqq k(t), k_{g}(t)$ coincides on compact sets with $t$ and by definition $k_{g}(t)$ is the largest group topology with this property. If $(G, t)$ is a $k$-group, then the identity map from $(G, t)$ to $\left(G, k_{g}(t)\right)$, which is a $k$-continuous homomorphism, is continuous, so $t=k_{g}(t)$. Conversely, suppose $t=k_{g}(t)$ and let $f$ be a $k$-continuous homomorphism on $G$. Let $t^{\prime}$ be the smallest

Received by the editors May 21, 1969 and, in revised form, October 27, 1969.

(1) This paper is based on the fifth chapter of the author's doctoral dissertation, which was written at the University of Rochester under the direction of Professor W. W. Comfort.

Copyright (C) 1970, American Mathematical Society 
topology on $G$ which is greater than or equal to $t$ and which makes $f$ continuous. Since $f$ is a homomorphism $t^{\prime}$ is a group topology, and since $f$ is $k$-continuous $t$ and $t^{\prime}$ coincide on compact sets. Hence $t^{\prime} \leqq k_{g}(t)=t$, so $f$ is continuous. Therefore $(G, t)$ is a $k$-group.

\subsection{Proposition. Each quotient group of a $k$-group is a $k$-group.}

Proof. If $g: G \rightarrow H$ is a quotient map and $h$ is a $k$-continuous homomorphism on $H$, then $h \circ g$ is a $k$-continuous homomorphism. If $G$ is a $k$-group, then $h \circ g$ is continuous and therefore $h$ is continuous.

Let $\left\{G_{\alpha}: \alpha \in A\right\}$ be a set of groups and let $H$ be a subgroup of $\prod_{\alpha \in A} G_{\alpha}$. Where for $B \subseteq A, \pi_{B}$ denotes the projection: $\prod_{\alpha \in A} G_{\alpha} \rightarrow \prod_{\alpha \in B} G_{\alpha} \times \prod_{\alpha \in A \mid B}\left\{e_{\alpha}\right\}, H$ is called invariant under projections if for each $B \subseteq A, \pi_{B} H \subseteq H$. In particular, $\prod_{\alpha \in A} G_{\alpha}$ and $\sum_{\alpha \in A} G_{\alpha}$ (the direct sum) are invariant under projections. The following result is established in [13, Theorem 5.7].

1.3. THEOREM. If $H$ is a subgroup of a product of $k$-groups, and $H$ is invariant under projections, then $H$ is a $k$-group.

We will use 1.3 to construct a pair of examples. Recall that a topological space $X$ is called a $k_{R}$-space if each $k$-continuous function from $X$ to $R$ (or equivalently any completely regular space $Z$ ) is continuous. It is easily shown that a $T_{0}$ topological group is a $k$-group if and only if each $k$-continuous homomorphism with $T_{0}$ range is continuous. Since $T_{0}$ groups are in fact completely regular Hausdorff, it follows that a $T_{0}$ group which is a $k_{R}$-space is a $k$-group.

1.4. ExAmple. There exists a $T_{0} k$-group which is not a $k_{R}$-space (and hence not a $k$-space).

Proof. In [17] Warner gives a topological group $G$ which is a $k$-space, is not locally compact and for which $C(G)$ with the compact-open topology is metrizable, hence a $k$-space. Under pointwise addition, $C(G)$ is a topological group. By 1.3, $G \times C(G)$ is a $k$-group. Where $e$ is the evaluation map, $e(g, f)=f(g), e$ is $k$-continuous but not continuous. (It is shown in [1] that for $e$ to be continuous, $G$ must be locally compact.) Thus $G \times C(G)$ is not a $k_{R}$-space.

For use in our next example and in $\S 3$, we recall the following result established by Weil in $[18, \S 5]$.

1.5. THEOREM. Let $G$ be a $T_{0}$, abelian topological group which is complete in the group uniformity, and suppose that $G$ has a base $\left\{H_{\alpha}\right\}$ at the identity consisting of open subgroups. Then $G$ is topologically isomorphic to the inverse limit of the quotient groups $\left\{G / H_{\alpha}\right\}$.

1.6. ExAmple. A closed subgroup of a $k$-group need not be a $k$-group.

Proof. Let $\Omega$ be the first uncountable ordinal and for each $\alpha$ in $\Omega$ let $G_{\alpha}$ be the discrete group $\{0,1\}$. Let $G$ be the direct sum of $\left\{G_{\alpha}: \alpha \in \Omega\right\}$ and for each $\alpha$ in $\Omega$ let $H_{\alpha}$ be the subgroup of $G$ consisting of those elements whose $\beta$ th coordinate is 
0 for each $\beta$ less than $\alpha$. Topologize $G$ by requiring that each translate of each $H_{\alpha}$ be open; with this topology $G$ is a nondiscrete topological group and the open subgroups $\left\{H_{\alpha}: \alpha \in \Omega\right\}$ form a base at the identity. Since any countable intersection of these subgroups is open, the identity is a $P$-point and hence, by homogeneity, $G$ is a $P$-space.

Now let $\left\{g^{\lambda}\right\}$ be a Cauchy net in $G$ and for each $\alpha$, let $h_{\alpha}$ be the limit in $G_{\alpha}$ of the Cauchy net $\left\{g_{\alpha}^{\lambda}\right\}$. If infinitely many of the $h_{\alpha}$ are not 0 , then there exists a $\beta$ in $\Omega$ such that $h_{\alpha}$ is not 0 for infinitely many $\alpha$ less than $\beta$. But since $\left\{g^{\lambda}\right\}$ is Cauchy, there exists a $\lambda_{0}$ such that $\lambda \geqq \lambda_{0}$ implies $g^{\lambda}$ is in $H_{\beta}+g^{\lambda}$, so $g_{\alpha}^{\lambda}$ can differ from 0 for at most finitely many $\alpha$ less than $\beta$. This contradicts our choice of $\beta$, so all but finitely many of the $h_{\alpha}$ must be 0 . Clearly $\left\{g^{\lambda}\right\}$ converges to $\left(h_{\alpha}\right)$ which is in $G$; therefore, since $\left\{g^{\lambda}\right\}$ was any Cauchy net in $G, G$ is complete.

Since $G$ is complete, abelian, and has a base of open subgroups, $G$ is, by the theorem above, isomorphic to the inverse limit of $\left\{G / H_{\alpha}\right\}$, and hence $G$ is isomorphic to a closed subgroup of $K=\prod_{\alpha \in \Omega}\left(G / H_{\alpha}\right)$. But each $G / H_{\alpha}$ is countable and discrete, hence a $k$-group, so by $1.3 K$ is a $k$-group. Finally, the closed subgroup of $K$ isomorphic to $G$ is not a $k$-group-since $G$ is a $P$-space, each compact subset of $G$ is finite and hence $k_{g}(G)$ is discrete, and therefore $k_{g}(G) \neq G$.

In view of the two examples above, it is interesting to note that the $k$-extension of a group topology preserves many of its properties.

1.7. TheOREM. Let $(G, t)$ be a $k$-group and let $k$ denote the $k$-extension of $t$. Then $k$ is homogeneous and makes inversion a homeomorphism. Furthermore, for each subgroup $H$ of $G, H$ is $t$-open if and only if $H$ is $k$-open.

Proof. Since translates and inverses of compact sets are compact, the maps $y \rightarrow x y$ and $x \rightarrow x^{-1}$ are $k$-continuous, so $k$ is homogeneous and makes inversion continuous.

For the second part of the theorem we need only show that each $k$-open subgroup is $t$-open. To this end, let $H$ be any $k$-open subgroup of $G$ and let $t^{\prime}$ be the smallest topology greater than or equal to $t$ which contains all two-sided translates of $H$. A straightforward application of 4.5 of [5] shows that $t^{\prime}$ is a group topology and since each translate of $H$ is $k$-open (because $k$ is homogeneous), $t \leqq t^{\prime} \leqq k$. But since $t=k g(t)$ we have $t=t^{\prime}$, so $H$ is $t$-open.

1.8. Corollary. Let $H$ be an open subgroup of a group $G$. Then $H$ is a $k$-group if and only if $G$ is a k-group.

Proof. If $H$ is a $k$-group, then each $k$-continuous homomorphism on $G$ is continuous on $H$ and hence $H$ is open in $G$. Conversely, suppose $(G, t)$ is a $k$ group and let $t^{\prime}$ be the $k_{g}$-extension of the relative topology on $H$. Let $t^{\prime \prime}$ be the group topology on $G$ which makes $H$ an open subgroup and which agrees with $t^{\prime}$ on $H$. Now $t^{\prime \prime}$ is clearly greater than or equal to $t$. Also, since $H$, like any open subgroup, is closed, $k\left(t^{\prime}\right)$ is just the relative topology on $H$ of $k(t)$. Hence, since 
each translate of $H$ is $k(t)$-open, we have $t \leqq t^{\prime \prime} \leqq k(t)$. Thus, since $(G, t)$ is a $k$ group and $t^{\prime \prime}$ is a group topology, $t=t^{\prime \prime}$. Therefore $t^{\prime}$ is just the restriction of $t$ to $H$, so $H$ is a $k$-group.

2. Bounds on the second dual. Recall that a homomorphism from a group $G$ to the circle group $T$ is called a character of $G$, and the set of all continuous characters of $G$ endowed with the compact-open topology and with multiplication defined pointwise is a topological group called the character group, or the dual group, of $G$. The character group of a group $(G, t)$ is denoted by $(G, t)^{\wedge}$ or, when confusion is unlikely, by $G^{\wedge}$.

A group $G$ is said to satisfy duality if the evaluation map $\tau$ defined by the relation $\tau(x)(\chi)=\chi(x)$ is a topological isomorphism between $G$ and its second dual, $\left(G^{\wedge}\right)^{\wedge}$. It is clear that $\tau$ always maps $G$ into its second dual. A group $G^{\prime}$ is said to have sufficiently many characters if, for each pair of distinct points $x, y$ in $G$ there exists a character $\chi$ in $G^{\wedge}$ such that $\chi(x) \neq \chi(y)$; it is easily seen that $\tau$ is one-toone if and only if $G$ has sufficiently many characters, so groups which satisfy duality must have this property. Also, since the dual of any group is abelian, groups which satisfy duality must be abelian and it is easily shown that the map $\tau$ on an abelian group is a homomorphism. Since we are interested in finding conditions under which a group will satisfy duality, we now restrict our attention to groups which are abelian and have sufficiently many characters. (Note that since any subgroup of an abelian group which has sufficiently many characters must also have these two properties, the following convention does not restrict our freedom to hypothesize subgroups. Furthermore, since a group with sufficiently many characters is clearly Hausdorff, all hypothesized groups will be completely regular Hausdorff.)

CONVENTION. Henceforth, all hypothesized topological groups are assumed to be abelian and to have sufficiently many characters.

The classical result concerning group duality is the Pontrjagin-Van Kampen duality theorem which asserts that locally compact abelian groups satisfy duality. This theorem has been generalized in a number of ways. One of the nicest of these results is Theorem 4 of [7], which states that a product of groups which satisfy duality must also satisfy duality. Thus many groups which are not locally compact are known to satisfy duality. For further information on this subject the reader is referred to [5], [7], [8], [10], [11], [15] and [16]; for proofs of the statements made above, other than Kaplan's theorem of [7], the reader is referred to [5].

By virtue of our convention, to show that a group $G$ satisfies duality one has only to show that the map $\tau$ is open, continuous, and onto. The $k$-group concept is useful in this context because of the fact, which we now set out to prove, that when $G$ is a $k$-group, $\tau$ is continuous.

Let $G$ be a group and let $\mathscr{C}$ be a collection of compact subsets of $G$. If each compact subset of $G$ is contained in some member of $\mathscr{C}$, then $\mathscr{C}$ is said to be a cobase 
for $G$. For convenience, we will say that a collection of subsets of $G$ is a base for $G$ if it is a base for the neighborhood system at the identity of $G$. Finally, a symmetric, connected neighborhood of the identity, 1 , of the circle group $T$ is said to be limited if it is contained in $\{z:|z-1|<\sqrt{ } 3\}$.

Limited neighborhoods have the following useful property: If $U$ is a limited neighborhood of 1 and if $V$ is any symmetric connected neighborhood of 1 , then there exists an integer $N$ such that whenever $z$ is not in $V$, one of the points $z, z^{2}, \ldots, z^{N}$ is not in $U$. Furthermore, if $U$ is a limited neighborhood of 1 and if $\mathscr{C}$ is a cobase for $G$, then $\{P(K, U): K \in \mathscr{C}\}$ is a base for $G^{\wedge}$. (Here and henceforth, $P(S, F)$ denotes the set of continuous characters which map $S$ into $F$.) These two facts are proved in [7, 2.6 and 2.9]. Recall that a set $F$ of functions from $X$ to $Y$ is said to be evenly continuous if for each point $x$ in $X$, each point $y$ in $Y$, and each neighborhood $U$ of $y$, there exist neighborhoods $V$ of $y$ and $W$ of $x$ such that $f(W) \subseteq U$ whenever $f$ is in $F$ and $f(x)$ is in $V$.

2.1. Lemma. Let $G$ be a topological group, let $d$ be the discrete topology on $G$, and let $E$ be a subset of $(G, d)^{\wedge}$. The following conditions on $E$ are equivalent:

(i) $E$ is evenly continuous;

(ii) $\bigcap_{x \in E} \chi^{-1}(U)$ is a neighborhood of the identity in $G$ for each neighborhood $U$ of 1 .

(iii) $\bigcap_{x \in E} \chi^{-1}(U)$ is a neighborhood of the identity in $G$ for some limited neighborhood $U$ of 1 .

Proof. Suppose $E$ is evenly continuous and let $U$ be any neighborhood of 1 . By even continuity, there exist neighborhoods $V$ of the identity, $e$, in $G$ and $W$ of 1 such that $\chi(V) \subseteq U$ whenever $\chi(e) \in W$ and $\chi$ is in $E$. But $\chi(e)^{\prime}=1 \in V$ for every character $\chi$, so $V$ is contained in $\bigcap_{x \in E} \chi^{-1}(U)$. Therefore $\bigcap_{x \in E} \chi^{-1}(U)$ is a neighborhood of $e$.

The implication (ii) $\Rightarrow$ (iii) is trivial. Suppose finally that $U$ is a fixed limited neighborhood of 1 for which $\bigcap_{x \in E} \chi^{-1}(U)$ is a neighborhood of $e$. Given $x$ in $G$, $z$ in $T$, and a neighborhood $V$ of $z$, let $W$ be a symmetric connected neighborhood of 1 such that $W^{2}$ is contained in $z^{-1} V$, and let $N$ be an integer such that whenever $y$ is not in $W$ one of the points $y, y^{2}, \ldots, y^{N}$ is not contained in $U$. Finally, let $U^{\prime}$ be a neighborhood of $e$ such that $\left(U^{\prime}\right)^{N}$ is contained in $\bigcap_{x \in E} \chi^{-1}(U)$. It suffices to show that $\chi\left(x U^{\prime}\right)$ is contained in $V$ whenever $\chi$ is in $E$ and $\chi(x)$ is in $z W$, which we do by contradiction. Suppose there is a $\chi$ in $E$ and a point $y$ in $U$ such that $\chi(x)$ is in $z W$ but $\chi(x y)$ is not in $V$. Since $\chi(x)$ is in $z W$ and since $z W^{2} \subseteq z V, \chi(y)$ is not in $W$ so there exists a positive integer $n \leqq N$ for which $(\chi(y))^{n}=\chi\left(y^{n}\right)$ is not in $U$. But since $y^{n} \in\left(U^{\prime}\right)^{n} \subseteq\left(U^{\prime}\right)^{N} \subseteq \bigcap_{x \in E} \chi^{-1}(U)$, this is impossible. Therefore $E$ is evenly continuous and the lemma is proved.

It should be emphasized that in the lemma just proved we did not assume continuity for the members of $E$. Indeed, for the special case in which $E$ has only one member the lemma tells us that a character is continuous if and only if it maps 
some neighborhood of $e$ into a fixed limited neighborhood of 1. (This fact is Lemma 2.1 in [8].) Using the lemma above and the fact, mentioned earlier, that a single limited neighborhood of 1 can be used to obtain a local base for the compact-open topology on a dual group, we will be able to carry out most of our computations using a fixed limited neighborhood of 1 . For this reason we adopt the following notation.

Notation. Let $U_{0}$ be a fixed closed limited neighborhood of 1 in $T$. If $\mathscr{S}$ is a collection of subsets of a group $G$ let $\mathscr{S}^{\wedge}$ denote the collection $\left\{P\left(S, U_{0}\right): S \in \mathscr{S}\right\}$ of subsets of $G^{\wedge}$.

2.2. Lemma. Let $G$ be a topological group and let $\mathscr{B}$ be a base for $G$. Then each member of $\mathscr{B}^{\wedge}$ is compact. Furthermore, $\mathscr{B}^{\wedge}$ is a cobase for $G^{\wedge}$ if and only if the map $\tau$ from $G$ to $\left(G^{\wedge}\right)^{\wedge}$ is continuous.

Proof. Let $t$ be the topology on $G$ and let $k=k(t)$. Since $G$ and $(G, k)$ have the same compact sets, the topology on $G^{\wedge}$ is just its relative topology as a subset of $C((G, k), T)$. Thus, by an Ascoli Theorem of sufficient generality (see for instance [3], [14] or [12]), and the fact that every subset of $C((G, k), T)$ is bounded, a subset of $G^{\wedge}$ is compact if and only if it is closed in $C((G, k), T)$ and evenly continuous on $(G, k)$. By the lemma just proved, each set in $\mathscr{B}^{\wedge}$ is evenly continuous on $G$ and hence on $(G, k)$. Also, if $f$ is the limit of a net of continuous homomorphisms each of which maps a fixed neighborhood $U$ of the identity into the closed set $U_{0}$, then $f$ is itself a homomorphism which maps $U$ into $U_{0}$, so by the lemma above $f$ is continuous. Thus each set in $\mathscr{B}^{\wedge}$ is closed in $C((G, k), T)$, and therefore each set in $\mathscr{B}^{\wedge}$ is compact.

If $\mathscr{B}^{\wedge}$ is a cobase for $G^{\wedge}$ then $\left(\mathscr{B}^{\wedge}\right)^{\wedge}$ is a base for $\left(G^{\wedge}\right)^{\wedge}$. But then, since $\tau(U)$ $\subseteq P\left(P\left(U, U_{0}\right), U_{0}\right), \tau$ is continuous at the identity and is therefore continuous. Conversely, suppose $\tau$ is continuous and let $K$ be any compact set in $G^{\wedge}$. Let $U=\bigcap_{x \in K} \chi^{-1}\left(U_{0}\right)$ and note that, since $\tau(U)=P\left(K, U_{0}\right) \cap \tau(G)$, the set $U$ is a neighborhood of the identity. Since $\mathscr{B}$ is a base, there exists a set $V$ in $\mathscr{B}$ such that $V \subseteq U$. But then $K \subseteq P\left(U, U_{0}\right) \subseteq P\left(V, U_{0}\right) \in \mathscr{B}^{\wedge}$. Therefore the collection $\mathscr{B}^{\wedge}$ is a cobase for $G^{\wedge}$.

2.3. THEOREM. If $G$ is a $k$-group, then the map $\tau$ from $G$ to $\left(G^{\wedge}\right)^{\wedge}$ is continuous.

Proof. Let $\mathscr{B}$ be the collection of neighborhoods of the identity in $G$; by the lemma above it suffices to show that $\mathscr{B}^{\wedge}$ is a cobase for $G^{\wedge}$, and to show this it suffices to show that $\bigcap_{x \in K} \chi^{-1}\left(U_{0}\right)$ is in $\mathscr{B}$ for each compact subset $K$ of $G^{\wedge}$. Let $t$ be the topology on $G$ and let $t^{\prime}$ be the smallest topology greater than or equal to $t$ which makes translations continuous and which makes each set of the form $\bigcap_{x \in K} \chi^{-1}\left(U_{0}\right)$, for $K$ a compact subset of $G^{\wedge}$, a neighborhood of the identity. Since, again by an appropriate Ascoli Theorem, each compact subset of $G^{\wedge}$ is evenly continuous on $(G, k(t))$, and since, by $1.7, k(t)$ is homogeneous, the topology $t^{\prime}$ is less than or equal to $k(t)$, so $t$ and $t^{\prime}$ have the same compact sets. But since $t^{\prime}$ is 
the relative topology on $G$ when $G$ is considered as a subgroup of its second dual, $t^{\prime}$ is a group topology. Therefore, since $(G, t)$ is a $k$-group, $t=t^{\prime}$ and the proof is complete.

Let $(G, t)$ be a topological group, let $G^{\wedge}$ be the set of continuous characters on $(G, t)$ and let $b(G)=\left(G^{\wedge}, d\right)^{\wedge}$ where $d$ is the discrete topology. The compact group $b(G)$ is called the Bohr compactification of $G$ (despite the fact that it is not in general a compactification of $G$ ). Also, let $b(t)$ denote the smallest topology on $G$ which makes each character in $G^{\wedge}$ continuous.

2.4. Theorem. Let $(G, t)$ be a topological group, let $\left(G^{\wedge}\right)^{\wedge}$ be the second dual of $(G, t)$ considered as a set, and let $t^{\prime \prime}$ be the relative topology on $G$ where $G$ is considered as a subgroup of the second dual of $(G, t)$. Then the following relations hold:

(i) $G \subseteq\left(G^{\wedge}\right)^{\wedge} \subseteq b(G)$;

(ii) $b(t) \leqq t^{\prime \prime} \leqq k_{g}(t)$.

Proof. Since $\tau$ is one-to-one and onto and since $b(G)$ is the set of all characters on $(G, t)^{\wedge}$, the relation (i) is clear. That $b(t) \leqq t$ follows from the fact, proved in [4], that $b(t)$ is just the relative topology on $G$ when $G$ is considered as a subgroup of $b(G)$. (For if $t^{\prime}$ is the topology on the dual of $(G, t)$, then since $t^{\prime}$ is less than or equal to $d$, the topology on $b(G)$, when restricted to $\left(G^{\wedge}\right)^{\wedge}$, is smaller than the topology which $\left(G^{\wedge}\right)^{\wedge}$ receives as the second dual of $(G, t)$. Restricting both of these topologies to the subgroup $G$, we have that $b(t)$ is less than or equal to $t^{\prime \prime}$.)

It remains to show that $t^{\prime \prime}$ is less than or equal to $k_{g}(t)$. Let $k^{\prime \prime}$ denote the relative topology on $G$ when $G$ is considered as a subgroup of the second dual of $\left(G, k_{g}(t)\right)$ and let $\mathscr{C}$ be the collection of compact subsets of the dual of $\left(G, k_{g}(t)\right)$. Then $\left\{U \cap G: U \in \mathscr{C}^{\wedge}\right\}$ is a base for the $k^{\prime \prime}$-neighborhood system at the identity. Similarly, if $\mathscr{C}^{\prime}$ is the collection of compact subsets of the dual of $(G, t)$, then $\left\{U \cap G: U \in\left(\mathscr{C}^{\prime}\right)^{\wedge}\right\}$ is a base for the $t^{\prime \prime}$-neighborhood system at the identity. But since $t$ and $k_{g}(t)$ have the same compact sets, $(G, t)$ is, under the natural identification, a topological subgroup of $\left(G, k_{g}(t)\right)$, so $\mathscr{C}^{\prime}$ is contained in $\mathscr{C}$. It follows that $t^{\prime \prime}$ is less than or equal to $k^{\prime \prime}$. But by the theorem above, $k^{\prime \prime}$ is less than or equal to $k_{g}(t)$; therefore $t^{\prime \prime}$ is less than or equal to $k_{g}(t)$.

Note that the bounds in 2.4 are "best" in the sense that they can all be achieved -for example, with $(G, t)$ the circle group, $G=b(G)$ and $b(t)=k_{g}(t)$. We close this section, on an irrelevant note, with an application of 2.2. A topological space is said to be hemicompact (or denumerable at infinity) if it has a countable cobase, and a locally compact space is hemicompact if and only if it is $\sigma$-compact. Taking this fact into account, a well-known theorem (see for instance 24.48 of [5]) states that, for $G$ locally compact, $G$ is first countable if and only if $G^{\wedge}$ is hemicompact; and dually, $G$ is hemicompact if and only if $G^{\wedge}$ is first countable. Lemma 2.2 yields the following generalization of this result.

2.5. THEOREM. If $G$ is a group which satisfies duality, then $G$ is first countable if 
and only if $G^{\wedge}$ is hemicompact; and dually, $G$ is hemicompact if and only if $G^{\wedge}$ is first countable. In general, if $G$ is first countable then $G^{\wedge}$ is hemicompact; and if $G$ is hemicompact then $G^{\wedge}$ is first countable.

This theorem can, of course, be extended in the obvious way so as to deal with bases' and cobases of any fixed cardinality. Examples are known which show that $G$ need not be first countable when $G^{\wedge}$ is hemicompact. We know of no examples which show that $G$ need not be hemicompact when $G^{\wedge}$ is first countable.

3. Duality. Let $H$ be a subgroup of a group $G$. If for each point $x$ in $G \backslash H$ there exists a character $\chi$ in $G^{\wedge}$ such that $\chi(H) \subseteq\{1\}$ and $\chi(x) \neq 1$, then $H$ is said to be dual-closed. If each continuous character on $H$ admits a continuous extension to a character on $G$, then $H$ is said to be dual-embedded.

It is clear that a dual-closed subgroup must be closed, and it is well known that every closed subgroup of a locally compact group is both dual-closed and dualembedded. Kaplan shows in Theorems 1 and 2 of [8] that every closed subgroup of a product of locally compact groups also has these two properties. (Indeed, his proofs show that the properties "every closed subgroup is dual-closed" and "every closed subgroup is dual-embedded" are each preserved under arbitrary products.)

3.1. THEOREM. Let $H$ be a dual-closed dual-embedded subgroup of a group for which $\tau$ is open and onto. If $H$ is a $k$-group, then $H$ satisfies duality.

Proof. Let $p: G^{\wedge} \rightarrow H^{\wedge}$ be the map $p(\chi)=\left.\chi\right|_{H}$; since $H$ is dual-embedded, $p$ is onto and since $p^{-1}\left(P\left(K, U_{0}\right)\right)=P\left(K, U_{0}\right)$ for compact $K \subseteq H, p$ is continuous. Now define $i:\left(H^{\wedge}\right)^{\wedge} \rightarrow\left(G^{\wedge}\right)^{\wedge}$ by the rule $i(\psi)=\psi \circ p$. Also let $\tau$ and $\tau_{H}$ denote the identity maps: $G \rightarrow G^{\wedge \wedge}$ and $: H \rightarrow H^{\wedge \wedge}$ respectively, and note that $\tau=i \circ \tau_{H}$. Now $\tau H=\left\{\psi \in\left(G^{\wedge}\right)^{\wedge}\right.$ : If $\chi \in G^{\wedge}$ and $\chi(H)=1$, then $\left.\psi(\chi)=1\right\}$, since $H$ is dual-closed. (If $x$ is in $G \backslash H$ there exists a character $\chi$ in $\hat{G}$ such that $\chi(H)=1$ but $\chi(x) \neq 1$ so $\tau(x)(\chi) \neq 1$.) Thus $i\left(\left(H^{\wedge}\right)^{\wedge}\right) \subseteq \tau H$ and therefore, since $H$ has sufficiently many characters, $i\left(\left(H^{\wedge}\right)^{\wedge}\right)=\tau H$. Thus $\tau_{H}$ is onto. To see that $\tau_{H}$ is open it suffices to note that $i$ is continuous: For $K \subseteq \hat{G}$ compact, $i^{-1}\left(P\left(K, U_{0}\right)\right)=P\left(p K, U_{0}\right)$ which is open since $p K$ is compact. Finally, $\tau_{H}$ is continuous by Theorem 2.3.

3.2. COROLlARY. If $G$ is a complete $k$-group which admits a base consisting of open subgroups, then $G$ satisfies duality.

Proof. By Theorem 1.5, the group $G$ is isomorphic to the inverse limit of its discrete factor groups, and hence is isomorphic to a closed subgroup of a product of locally compact groups. Since, by Theorems 1 and 2 of [8] every such subgroup is dual-closed and dual-embedded, and since by Theorem 4 of [7] each product of locally compact groups satisfies duality, the hypotheses of the theorem apply to $G$. Therefore $G$ satisfies duality.

The lemma which follows is well known in the case where $G$ is locally compact. 
We include the details of its proof to assure the reader that it remains valid without restriction on $G$.

3.3. Lemma. If $H$ is an open subgroup of a group $G$, then $H$ is dual-closed and dual-embedded in $G$.

Proof. Since $G / H$ is discrete it has sufficiently many characters. Thus, for any point $x$ in $G \backslash H$ there exists a character $\chi$ in $(G / H)^{\wedge}$ such that $\chi(x H) \neq 1$. Define $\psi: G \rightarrow T$ by the relation $\psi(y)=\chi(y H)$. Then $\psi$ is clearly a homomorphism, and since it is continuous on the open subgroup $H$ it is continuous. Therefore $H$ is dual-closed.

Now let $\chi$ be a continuous character on $H$. Since $T$ is divisible, $\chi$ can be extended to a homomorphism $\chi^{\prime}$ from $G$ into $T$ (see, for instance, Theorem A.7 of [5]). Since $\chi^{\prime}$ is continuous on $H$, it is continuous. Therefore $H$ is dual-embedded.

3.4. COROLlaRY. If $H$ is an open subgroup of a $k$-group which satisfies duality, then $H$ satisfies duality.

Proof. By 1.8 and 3.3, $H$ satisfies the hypothesis of Theorem 3.1.

3.5. COROLlARY. Every closed subgroup of a countable product of locally compact groups satisfies duality.

Proof. A countable product of locally compact groups is a $k$-space (see [2] or [13]). Thus every closed subgroup of such a product is a $k$-space and therefore a $k$-group. Therefore, as in the proof of 3.2 the hypotheses of Theorem 3.1 are satisfied.

Corollary 3.5 generalizes the principal result of [8] which states that a certain closed subgroup of a countable product of locally compact groups (an inverse limit of a sequence of locally compact groups) satisfies duality. That a closed subgroup of an arbitrary product of locally compact groups need not satisfy duality is shown in [11] by Leptin by an example which is, in effect, the group $G$ in Example 1.6. Every countably compact locally compact (indeed, every countably compact complete) group is compact, and a product space is a $k$-space only if all but countably many of its factors are countably compact (see [13]). Thus except for the trivial case in which all but countably many of the factors are compact, the proof of Corollary 3.5 does not generalize to any uncountable products of locally compact groups.

We will now give several examples to demonstrate some of the relationships between $k$-groups and groups which satisfy duality. There are, however, several questions which we have not been able to answer, the most interesting of which appears to be: Does every complete $k$-group (which is abelian and has sufficiently many characters) satisfy duality?

EXAMPLES. (i) A complete abelian $k$-group need not have sufficiently many characters. 
(ii) A closed subgroup of a complete $k$-group which satisfies duality need not be dual-closed.

(iii) A group which satisfies duality need not be a $k$-group. (Indeed, such a group need not be complete.)

(iv) There exists a $k$-group (which is not complete) which does not satisfy duality.

Proof. (i) Hewitt and Ross in [5, p. 371] give an example of a complete abelian metrizable group which is infinite but which has only one continuous character. Since metrizable groups are $k$-groups, this shows that a complete abelian metrizable $k$-group need not have sufficiently many characters.

(ii) R. Hooper gives an example in [6] of a subgroup of a real Banach space which is not dual-closed. M. Smith shows in [15] that each real Banach space, when considered as an additive group, satisfies duality. Since Banach spaces are complete and metrizable, Hooper's example shows that a closed subgroup of a complete $k$-group which satisfies duality need not be dual-closed. (In speaking of completeness for linear spaces and for topological groups we are, formally, speaking of two different concepts: A linear space is complete if it is complete with respect to the usual linear space uniform structure and an abelian group is complete if it complete with respect to the usual group uniform structure. However, when $G$ is a linear space considered as an additive group these two uniformities, and hence these two forms of completeness, are the same.)

(iii) In [9] Kōmura gives an example of a real linear space which is reflexive but not complete, and in [15] Smith shows that each reflexive real linear space, considered as an additive group, satisfies duality. Thus Kōmura's example shows that a group which satisfies duality need not be complete. Now it is clear that the dual of a $k$-group is complete. Thus if $G$ is a group which satisfies duality and if $G$ is not complete, then $G^{\wedge}$, which also satisfies duality, is not a $k$-group. Therefore, a group which satisfies duality need not be a $k$-group.

(iv) Let $G$ be the additive group $Q / Z$ with the quotient topology, where $Q$ is the group of rational numbers and $Z$ the group of integers. Since $G$ is metrizable it is a $k$-group, so it suffices to show that $G$ does not satisfy duality. Since the completion of $G$ is $R / Z$ which is isomorphic to $T, G^{\wedge}$ is equal, as a set, to $T^{\wedge}$, which is equated with $Z$ under the rule $n \rightarrow \chi_{n}$, where $\chi_{n}(q)=\exp ($ inq). Let $K=\{1 / n: n=1,2, \ldots\}$ and let $U$ be an open neighborhood of the identity in $T$ which does not contain $\exp \left(\frac{1}{2} i\right)$. Since the subset $K$ of $G$ is a sequence which converges to the point $0, K$ is compact and hence $P(K, U)$ is open in $G^{\wedge}$. But for $n \neq 0$ we have $\chi_{n}(1 / 2 n)=$ $\exp \left(\frac{1}{2} i\right) \notin U$, so $P(K, U)=\left\{\chi_{0}\right\}$. Thus $G$ is discrete so $\left(G^{\wedge}\right)^{\wedge}$ is just $Z^{\wedge}$ which is $T$. Therefore $G$ does not satisfy duality.

\section{REFERENCES}

1. R. Arens, A topology for spaces of transformations, Ann. of Math. (2) 47 (1946), 480-495. MR 8, 165. 
2. A. Arhangel'skiĭ, On a class of spaces containing all metric and all locally bicompact spaces, Dokl. Akad. Nauk SSSR 151 (1963), 751-754=Soviet Math. Dokl. 4 (1963), 1051-1055. MR 27 \#2959.

3. R. W. Bagley and J. S. Yang, On k-spaces and function spaces, Proc. Amer. Math. Soc. 17 (1966), 703-705. MR 33 \#693.

4. W. W. Comfort and K. A. Ross, Topologies induced by groups of characters, Fund. Math. 55 (1964), 283-291. MR 30 \#183.

5. E. Hewitt and K. A. Ross, Abstract harmonic analysis. Vol. I: Structure of topological groups. Integration theory, group representations, Die Grundlehren der math. Wissenschaften, Band 115, Academic Press, New York and Springer-Verlag, Berlin and New York, 1963. MR 28 \#158.

6. R. Hooper, A study of topological Abelian groups based on normed space theory, Doctoral Dissertation, Univ. of Maryland, College Park, 1967.

7. S. Kaplan, Extensions of the Pontrjagin duality. I: Infinite products, Duke Math. J. 15 (1948), 649-658. MR 10, 233.

8. - Extensions of the Pontrjagin duality. II: Direct and inverse sequences, Duke Math. J. 17 (1950), 419-435. MR 14, 245.

9. Y. Kōmura, Some examples on linear topological spaces, Math. Ann. 153 (1964), 150-162. MR 32 \#2884.

10. H. Leptin, Bemerkung zu einem Satz von S. Kaplan, Arch. Math. 6 (1955), 139-144. MR 16, 568.

11. — Zur Dualitätstheorie projektiver Limites abelscher Gruppen, Abh. Math. Sem. Univ. Hamburg 19 (1955), 264-268. MR 16, 899.

12. N. Noble, Ascoli theorems and the exponential map, Trans. Amer. Math. Soc. 143 (1969), 393-411.

13. - The continuity of functions on cartesian products, Trans. Amer. Math. Soc. 149 (1970), 187-198.

14. H. Poppe, Stetige Konvergenz und der Satz von Ascoli und Arzelà, Math. Nachr. 30 (1965), 87-122. MR 32 \#6400.

15. M. Smith, The Pontrjagin duality theorem in linear spaces, Ann. of Math. (2) 56 (1952), 248-253. MR 14, 183.

16. N. Vilenkin, Direct spectra of topological Abelian groups and their limit groups, Dokl. Akad. Nauk SSSR 72 (1950), 617-620. (Russian) MR 12, 79.

17. S. Warner, The topology of compact convergence on continuous function spaces, Duke Math. J. 25 (1958), 265-282. MR 21 \#1521.

18. A. Weil, L'intégration dans les groupes topologiques et ses applications, Actualités Sci. Indust., nos. 869, 1145, Hermann, Paris, 1940, 1951. MR 3, 198.

\section{Clark University,}

WORCESTER, MASSACHUSETTS 01610

UNIVERSITY OF WASHINGTON,

SeattLe, Washington 98105 